University of Wollongong

Research Online

Faculty of Engineering - Papers (Archive)

Faculty of Engineering and Information

Sciences

March 2005

\title{
Magneto-optical far-infrared absorption spectroscopy of the hole states of indium phosphide
}

\author{
R. A. Lewis \\ University of Wollongong, roger@uow.edu.au \\ Y. Wang \\ Florida State University, USA
}

Follow this and additional works at: https://ro.uow.edu.au/engpapers

Part of the Engineering Commons

https://ro.uow.edu.au/engpapers/225

\section{Recommended Citation}

Lewis, R. A. and Wang, Y.: Magneto-optical far-infrared absorption spectroscopy of the hole states of indium phosphide 2005.

https://ro.uow.edu.au/engpapers/225

Research Online is the open access institutional repository for the University of Wollongong. For further information contact the UOW Library: research-pubs@uow.edu.au 


\title{
Magneto-optical far-infrared absorption spectroscopy of the hole states of indium phosphide
}

\author{
R. A. Lewis \\ Institute for Superconducting and Electronic Materials, University of Wollongong, Wollongong NSW 2522, Australia \\ Y.-J. Wang \\ National High Magnetic Field Laboratory at Florida State University, Tallahassee, Florida 32310, USA \\ (Received 1 November 2004; published 29 March 2005)
}

\begin{abstract}
Far-infrared absorption spectroscopy in magnetic fields of up to $30 \mathrm{~T}$ of the zinc acceptor impurity in indium phosphide has revealed for the first time a series of free-hole transitions (Landau-related series) in addition to the familiar bound-hole transitions (Lyman series) as well as hitherto unobserved phonon replicas of both series. Analysis of these data permits the simultaneous direct experimental determination of (i) the hole effective mass, (ii) the species-specific binding energy of the acceptor impurity, (iii) the absolute energy levels of the acceptor excited states of both odd and even parity, (iv) more reliable, and in some cases the only, $g$ factors for acceptor states, through relaxation of the selection rules for phonon replicas, and (v) the LO phonon energy. The method is applicable to other semiconductors and may lead to the reappraisal of their physical parameters.
\end{abstract}

DOI: 10.1103/PhysRevB.71.115211

PACS number(s): 75.47.Gk, 78.30.-j, 63.20.Dj

\section{INTRODUCTION}

InP is a direct-gap $\epsilon_{g}=1.42 \mathrm{eV}$ III-V semiconductor alloy of tremendous technological importance in photonics (photonics integrated circuits, laser diodes, light-emitting diodes, photodetectors, quantum-dot lasers, optical modulators), electronics (high-speed, high-power applications, highelectron-mobility transistors, [double] heterojunction bipolar transistors), and radiation detection (medical physics, solar cells, neutrino physics). Despite this, knowledge of the valence band and hole states remains relatively poor. The chief $p$-type impurity deliberately incorporated into InP is $\mathrm{Zn}$. In this work, InP:Zn is examined in magnetic field to clarify the appraisal of the parameters of hole-doped semiconductors.

The ground-state (binding, ionization) energy $E_{A}$ of a generic acceptor in InP has been calculated by Baldereschi and Lipari within a spherical model, ${ }^{1}$ including cubic contributions $^{2}$ and inversion asymmetry. ${ }^{3}$ Wang and Chen have calculated $E_{A}$ for a generic acceptor in InP using a $\mathbf{k} \cdot \mathbf{p}$ Hamiltonian. ${ }^{4}$ They include a central-cell potential correction to obtain the energy of $\mathrm{Zn}$ impurity. Results of these calculations are summarized in Table I. It is notable that the $\mathrm{Zn}$ specific calculation differs substantially from each of the generic calculations, underscoring the importance of the central-cell correction, which is not easy to calculate with precision. Experimental values of $E_{A}$ have been determined by photoluminescence (PL) and also appear in Table I. These differ among themselves by $4.5 \mathrm{meV}$ (about 10\%).

Excited states of shallow substitutional acceptors in tetrahedrally bonded semiconductors inherit the complex character of the valence band. Starting from a spherical model, ${ }^{1}$ the inclusion of spin-orbit coupling requires atomic $S$ states be denoted $S_{3 / 2}$; the subscript gives the total angular momentum $F$. The $P$ states become $P_{1 / 2}, P_{3 / 2}$, and $P_{5 / 2}$. Adding firstorder cubic terms to the Hamiltonian ${ }^{2}$ affects the $2 P_{5 / 2}$ state, which now splits into $2 P_{5 / 2}\left(\Gamma_{8}^{-}\right)$and $2 P_{5 / 2}\left(\Gamma_{7}^{-}\right)$, where the terms $\Gamma_{k}^{\pi}$ refer to irreducible representations of the $\mathrm{O}_{h}$ group.
In general, any state may be labeled as $n \Gamma_{k}^{\pi}$ of $\mathrm{O}_{h}$ where $\pi$ is the parity, denoted + or,$- k$ takes on the values 6,7 , or 8 , and $n=1,2,3, \ldots$ is the sequence number for states of a given $\pi$ and $k$ in order of energy. While the symbol $n \Gamma_{k}^{\pi}$ completely specifies the state it is sometimes convenient to retain the atomic notation and, for example, write the ground state as $1 S_{3 / 2}\left(1 \Gamma_{8}^{+}\right)$and the first odd-parity excited state as $2 P_{3 / 2}\left(1 \Gamma_{8}^{-}\right)$. The energies of excited states have not been subject to as great theoretical or experimental investigation as has the ground state; some limited values are given in Table II. Not only the energies of the states themselves, but even energy differences between states exhibit considerable variation in Table II. For example, the $1 \Gamma_{8}^{+} / 1 \Gamma_{8}^{-}$difference is given variously as $19.31,20.72$, and $24.5 \mathrm{meV}$, a discrepancy much larger than the resolution of typical spectrometers.

Transition energies (energy differences between states) have been measured by electronic Raman scattering (ERS) ${ }^{10}$ and by absorption spectroscopy (described here). Such methods do not yield directly the energies of the states them-

TABLE I. Ground-state $\left(1 \Gamma_{8}^{+}\right)$energy $E_{A}$ of InP:Zn.

\begin{tabular}{lcc}
\hline \hline \multicolumn{1}{c}{ Theoretical model } & $\mathrm{meV}$ & $\mathrm{cm}^{-1}$ \\
\hline Spherical model, generic (Ref. 1) & 35.2 & 283.9 \\
- including cubic terms (Ref. 2) & 35.20 & 283.90 \\
-, - including asymmetry (Ref. 3) & 36.3 & 292.8 \\
$\mathbf{k} \cdot \mathbf{p}$ Hamiltonian (Ref. 4) & 38.9 & 313.7 \\
-, with chemical correction for Zn (Ref. 4) & 47.2 & 380.7 \\
\hline
\end{tabular}

Experimental method

\begin{tabular}{lcc} 
PL, 4.2 K (Ref. 5) & $45.5 \pm 0.8$ & $367.0 \pm 6.5$ \\
PL (Ref. 6) & $46.1 \pm 0.6$ & $371.8 \pm 4.8$ \\
PL (Ref. 7) & 46.5 & 375.0 \\
PL (Ref. 8) & 47.0 & 379.1 \\
PL, 50 K (Ref. 9) & $50 \pm 2$ & $403 \pm 16$ \\
\hline \hline
\end{tabular}


TABLE II. Energies of some excited states of InP:Zn.

\begin{tabular}{lccc}
\hline \hline State & $\begin{array}{c}\text { Cubic model } \\
\text { (Ref. 2) } \\
\text { meV }\end{array}$ & $\begin{array}{c}\text { Cubic model } \\
\text { (Ref. 11) } \\
\text { meV }\end{array}$ & $\begin{array}{c}\text { PL } \\
\text { (Ref. 12) } \\
\text { meV }\end{array}$ \\
\hline $1 S_{3 / 2}\left(1 \Gamma_{8}^{+}\right)$ & 35.20 & 37.8 & 42 \\
$2 S_{3 / 2}\left(2 \Gamma_{8}^{+}\right)$ & 10.53 & & 14.4 \\
$2 P_{1 / 2}\left(1 \Gamma_{6}^{-}\right)$ & 1.97 & & \\
$2 P_{3 / 2}\left(1 \Gamma_{8}^{-}\right)$ & 15.89 & 17.08 & 17.5 \\
$2 P_{5 / 2}\left(2 \Gamma_{8}^{-}\right)$ & 9.98 & 10.68 & 12.8 \\
$2 P_{5 / 2}\left(1 \Gamma_{7}^{-}\right)$ & 7.32 & & 9.8 \\
\hline \hline
\end{tabular}

selves. PL and ERS are sensitive to transitions in which parity is conserved. Absorption spectroscopy is complementary, the dipole selection rules permitting transitions between states of different parity.

\section{EXPERIMENT}

The samples studied here were bulk InP doped with $\mathrm{Zn}$ at $1 \times 10^{17}$ atoms $\mathrm{cm}^{-3}$, wedged to suppress optical interference between front and back faces. A Bruker Model IFS 113v Fourier-transform infrared spectrometer equipped with a globar light source and liquid-helium-cooled Si bolometer detector was used. Measurements were made in a $17.5 \mathrm{~T}$ superconducting magnet and a $30 \mathrm{~T}$ resistive magnet. The light was conducted to the sample at field center via a metal light pipe and a condenser cone. The samples were aligned with a $\langle 100\rangle$ axis parallel to the field. This was also the direction of (unpolarized) light propagation (Faraday geometry); in this arrangement, $\boldsymbol{E} \perp \boldsymbol{B}$.

\section{RESULTS AND DISCUSSION}

Figure 1 shows the transmission of InP: $\mathrm{Zn}$ in the energy range $72-86 \mathrm{meV}$ for $B=0$ and $10<B<30 \mathrm{~T}$. Absorption

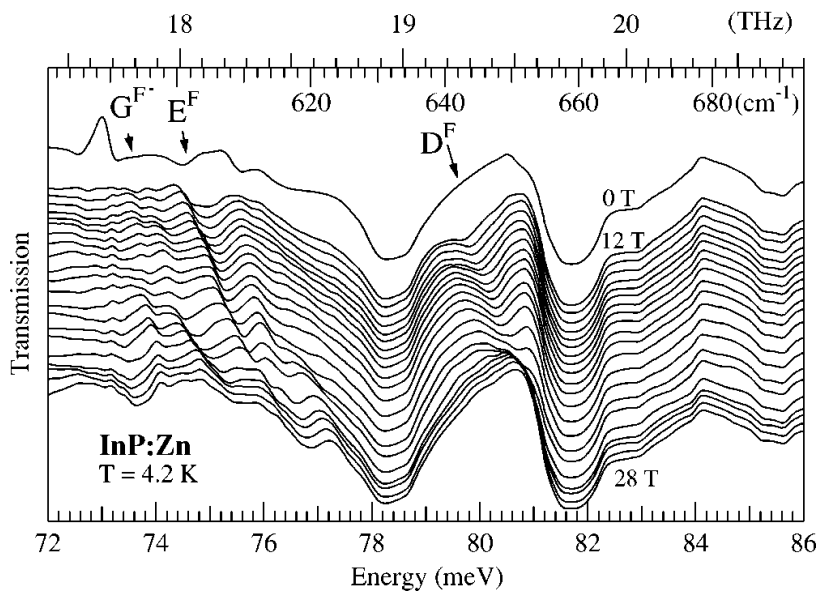

FIG. 1. Transmission of InP:Zn in the energy range $72-86 \mathrm{meV}$ for fields $B=0$ (topmost), 11.5, 12.5, 13.0, 13.5, 13.9, 14.9, 15.4, $15.9,16.3,17.1,17.3,18.0,19.2,20.4,21.6,22.8,25.2,26.4,27.0$, 27.6 , and $28.2 \mathrm{~T}$. features that move with field and are related to transitions from the $1 \Gamma_{8}^{+}$ground state to the excited states $1 \Gamma_{8}^{-}, 2 \Gamma_{8}^{+}$, and $2 \Gamma_{8}^{-}$are indicated, respectively, as $G^{F}, E^{F}$, and $D^{F}$. The superscripts signify that these features are not the interstate transitions themselves, but their phonon replicas in resonance with the valence band continuum, or Fano resonances. ${ }^{13}$

Fano resonances have been observed previously in many and various semiconductor systems. Here we restrict the discussion to the bound states of impurities. Fano resonances have been reported for shallow and deep donor and acceptor impurities in $\mathrm{Si}^{14-24}{ }^{14}$ donors $^{25}$ and acceptors ${ }^{26}$ in $\mathrm{Ge}, \mathrm{Mn}$ in $\mathrm{GaAs}^{27}$ and $\mathrm{Li}$ in $\mathrm{ZnSe}^{28}$

The Fano function $F(\varepsilon, q)=(q+\varepsilon)^{2} /\left(1+\varepsilon^{2}\right)$ involves the shape parameter $q$ and the dimensionless reduced energy

$$
\varepsilon=\frac{\hbar \omega-\left[E_{\mathrm{hole}}-\hbar \omega_{\text {phonon }}-\mathcal{F}\right]}{\Gamma / 2},
$$

where $\hbar \omega, E_{\text {hole }}$, and $\hbar \omega_{\text {phonon }}$ are, respectively, the photon, hole-transition, and phonon energy, $\mathcal{F}$ is the coupling parameter, and $\Gamma$ is the spectral width. The Fano parameters may be determined from the absorption spectrum by a simple construction. ${ }^{29}$ Here $q \gg \varepsilon$ and the spectral width is almost the same as for the parent transitions.

In magnetic field the degeneracy of the acceptor energy states is lifted, analogously to the Zeeman effect in atomic hydrogen. In discussing the effect of a magnetic field on different hydrogenic systems it is convenient to introduce a dimensionless unit for the strength of the magnetic field. This is conventionally taken to be the ratio of the magnetic energy $\mu_{B} B$ (where $\mu_{B}$ is the Bohr magneton and $B$ the magnetic field) to the Rydberg energy Ry. This ratio is unity at the magnetic field

$$
B_{0}=\frac{\mathrm{Ry}}{\mu_{B}},
$$

which has the value of 235052 T. Scaling for an acceptor having first Luttinger parameter $\gamma_{1}$ in a semiconductor host of dielectric constant $\epsilon$, the effective Rydberg

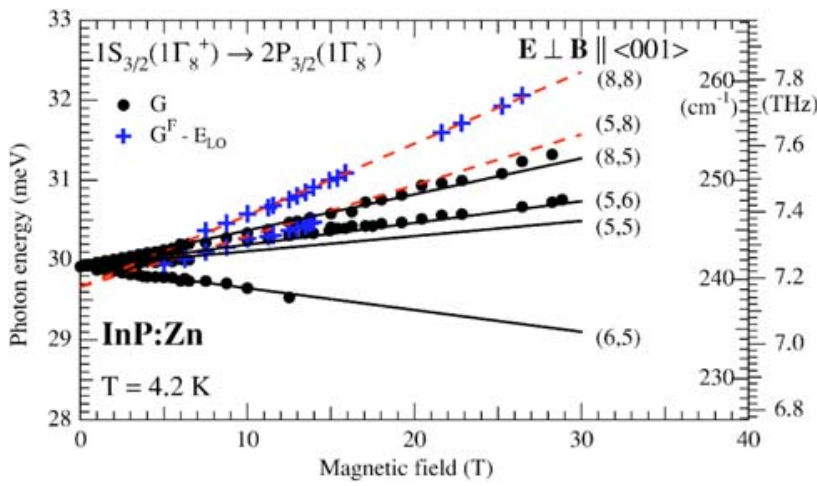

FIG. 2. (Color online) Field dependence of $1 \Gamma_{8}^{+} \rightarrow 1 \Gamma_{8}^{-}$transition $(G)$ and phonon replicas (Fano resonances $G^{F}$ ). The latter have been reduced in energy by $\hbar \omega_{\mathrm{LO}}$. The transitions are labeled according to the notation of Ref. 34. The lines are given by Eq. (2) using the $g$ factors stated in the text. 


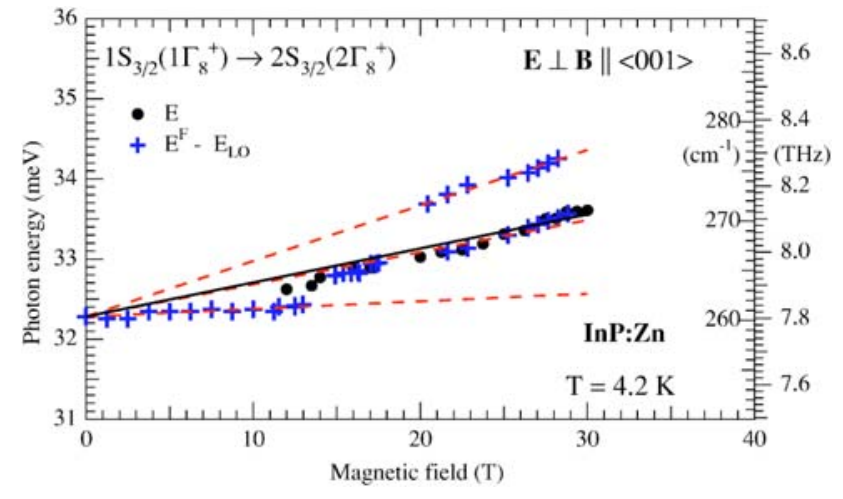

FIG. 3. (Color online) Field dependence of $1 \Gamma_{8}^{+} \rightarrow 2 \Gamma_{8}^{+}$transition $(E)$, formally forbidden in this geometry, and three phonon replicas $\left(E^{F}\right)$. The latter have been reduced in energy by $\hbar \omega_{\mathrm{LO}}$. The lines given are least-squares fits.

$\mathrm{Ry}^{*}=\mathrm{Ry} / \gamma_{1} \epsilon^{2}$ and the effective Bohr magneton $\mu_{B}^{*}=\gamma_{1} \mu_{B}$ and so

$$
B_{0}^{*}=\frac{1}{\gamma_{1}^{2} \epsilon^{2}} \frac{\mathrm{Ry}}{\mu_{B}} .
$$

Taking $\gamma_{1}$ to be 5.15 (Ref. 30) for InP, $B_{0}^{*}=58 \mathrm{~T}$. This magnetic field is also that at which the magnetic length $\sqrt{\hbar / e B}$ is the same as the Bohr radius $a_{0}$.

It is usual to refer to fields below $B_{0}^{*}$ as "low" and to fields above $B_{0}^{*}$ as "high." At the experimentally highest field $B=30 \mathrm{~T}$ employed here, $B_{0}^{*}<0.5$, and so in this sense all the fields employed here are "low." More precisely, our interest is to the extent that a theory of Zeeman splitting based on linear $g$ factors as developed via group theory by Bhattacharjee and Rodrigue ${ }^{31}$ is expected to be adequate to describe the results. It has been argued that at any field the atomic notation is not strictly correct and that the states should be labeled by the high-field (Landau) notation, but this would be an extreme view. It might be argued, on the basis that even the ground-state wavefunctions extend over many Bohr radii, ${ }^{32}$ that a better criterion for the onset of high field is when the magnetic length is not $a_{0}$, but rather, say, $10 a_{0}$. According to this argument, a high field would be only $B_{0}^{*} / 100$, or $\sim 0.6 \mathrm{~T}$ in InP and $\sim 0.3 \mathrm{~T}$ in GaAs. Such a criterion appears unduly conservative in light of the experimental data published for these systems. Schmitt et al., ${ }^{11}$ in a theoretical study, propose a rule of thumb that the linear terms are adequate in describing the states to about $B_{0}^{*} / 5$, or about $12 \mathrm{~T}$ in InP. However, it has been demonstrated in experiments in $p$-GaAs that the splittings of the magnetotransitions often follow a linear dependence to much higher fields and an explanation of that phenomenon given: ${ }^{33}$ often the quadratic term is the same for the two excited states and so is eliminated if the difference is calculated. This allows accurate calculation of $g$ factors using the linear approximation.

With magnetic field parallel to a $\langle 001\rangle$ crystal axis, the energies of the four sublevels of a $\Gamma_{8}$ state are given by

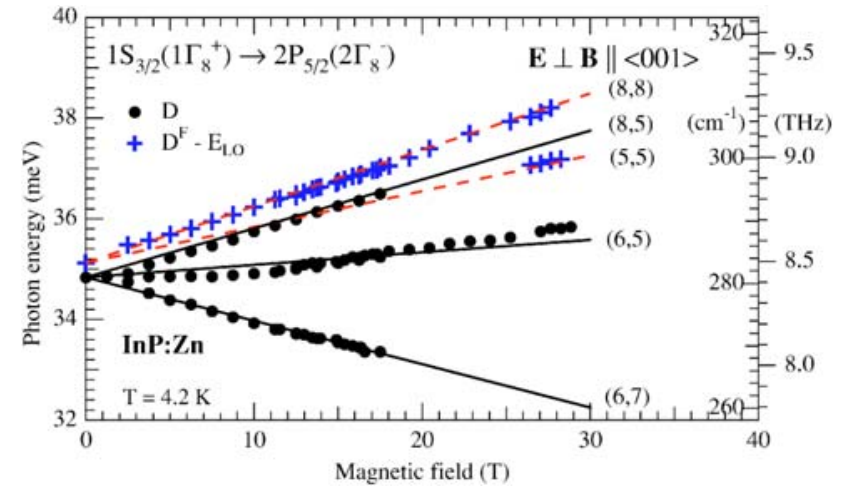

FIG. 4. (Color online) Field dependence of $1 \Gamma_{8}^{+} \rightarrow 2 \Gamma_{8}^{-}$transition (D) and phonon replicas (Fano resonances); the latter have been reduced in energy by $\hbar \omega_{\mathrm{LO}}$. The transitions are labeled according to the notation of Ref. 34. The lines are given by Eq. (2) using the $g$ factors stated in the text.

$$
E_{m}^{p}=\mu_{B}\left(m g_{1}^{p}+m^{3} g_{2}^{p}\right) B+\left[q_{1}^{p}+\left(q_{2}^{p}+q_{3}^{p}\right) m^{2}\right] B^{2},
$$

where $m= \pm \frac{1}{2}, \pm \frac{3}{2}$ is a spin quantum number characterizing the state, here given within the $T_{d}$ notation, the terms $g$ and $q$ are parameters of the material, and the label $p$ denotes the particular $\Gamma_{8}$ state.

Figure 2 gives the field dependence of the features associated with the transition $1 \Gamma_{8}^{+} \rightarrow 1 \Gamma_{8}^{-}(G$ line $)$ and its Fano resonances $\left(G^{F}\right)$. For convenience of comparison, the Fano resonances have been shifted down in energy by the energy of the LO phonon. The zero-field positions of the parent line and the Fano resonance do not precisely match; here the (unreduced) coupling parameter $\mathcal{F}_{G}=0.26 \mathrm{meV}$. The intensity of the lower-energy components decreases with magnetic field due to magnetic depopulation; only two components, those of high energy, are evident beyond $18 \mathrm{~T}$. The selection rules permit four parallel and two perpendicular components to be observed in the parent transition. The selection rules relax for phonon replicas. ${ }^{35} \mathrm{We}$ attribute the new, higher-energy $G^{F}$ component to a transition forbidden in the parent series for this polarization. The larger number of components now available allows us to reanalyze the splitting pattern and determine the $g$ factors of the $1 \Gamma_{8}^{+}$and $1 \Gamma_{8}^{-}$states with greater confidence than previously. We find that the ground state $1 \Gamma_{8}^{+}$has $g_{1}^{\prime}=+0.83 \pm 0.06$ and $g_{2}^{\prime}=-0.12 \pm 0.06$, and the excited state $1 \Gamma_{8}^{-}$has $g_{1}^{G}$ $=1.58 \pm 0.06$ and $g_{2}^{G}=-0.72 \pm 0.06$.

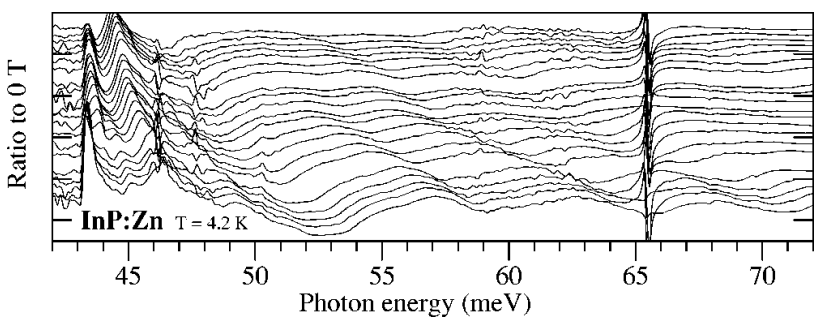

FIG. 5. Landau-related series of lines. Fields are as for Fig. 1 except zero field is omitted. 


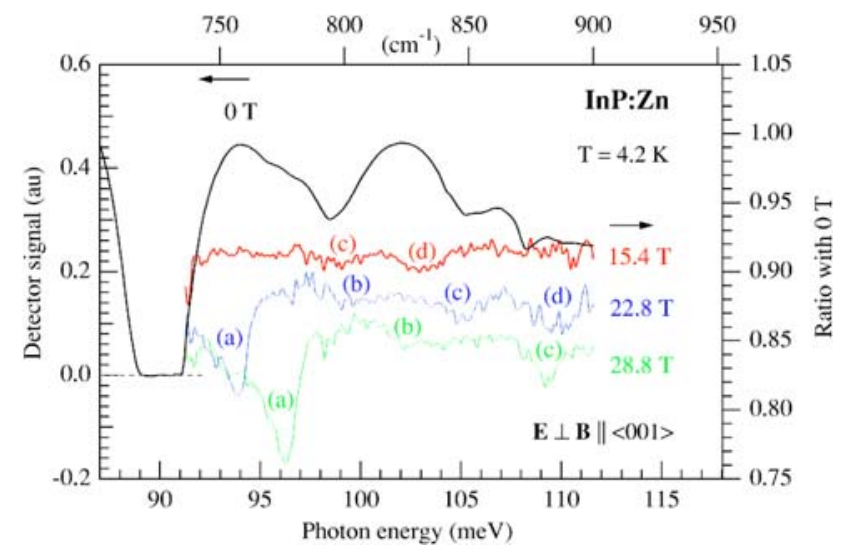

FIG. 6. (Color online) High-energy, high-field phonon replica of Landau-related series. Spectra at field are ratioed with zero field spectrum and then offset by $0.12,0.17$, and 0.20 , respectively, for 15.4, 22.8, and 28.8 T. Zero-field spectrum illustrates instrument blackout region and high-energy limit.

The $E$ transition $1 \Gamma_{8}^{+} \rightarrow 2 \Gamma_{8}^{+}$is formally forbidden by the selection rules for this geometry. Here the strong field induces wave-function overlap, permitting the observation of one component of the $E$ line in absorption, confirming the transition energy measured in ERS. Three phonon replicas are observed (Fig. 3). Even so, there are too few components to unravel the $g$ factors of the $2 \Gamma_{8}^{+}$state. For this transition, $\mathcal{F}_{\mathrm{E}}=0$. We speculate this is connected with both the initial and final states being of even parity.

At small fields (3 T), the $D$ transition $1 \Gamma_{8}^{+} \rightarrow 2 \Gamma_{8}^{-}$broadens and disappears. ${ }^{36}$ At higher fields it reemerges, leading to a reassignment of the unperturbed energy on the basis of magnetospectroscopy. ${ }^{34}$ Even so, the small number of observed components was insufficient to allow the determination of the $g$ factors of the $2 \Gamma_{8}^{-}$state. Here the effects of magnetic depopulation are observed for the $D$ line, as they were for the $G$. Only two components are observed beyond $12 \mathrm{~T}$, and only one beyond $18 \mathrm{~T}$. Now, with the Fano resonances included $\left(\mathcal{F}_{D}=-0.29 \mathrm{meV}\right)$, four components (one parallel) rather than three are evident (Fig. 4), and at last the $g$ factors of the excited state can be established. These are determined to be $g_{1}^{D}=2.38 \pm 0.08$ and $g_{2}^{D}=-0.81 \pm 0.08$.

ERS measurements have reported lines labeled $C, 1$, and 2, of energies 37.25, 39.51, and $40.99 \mathrm{meV}^{10,37}$ Such energies are not accessible to our technique, lying in the reststrahlen region, which extends from $\sim 38$ to 44 meV. In this range essentially all photons are reflected. We can access the phonon replica of this region-but no transitions are observed at the corresponding energies, even at field.

Above the reststrahlen band a new series of lines emerges. Three components have been observed earlier to $17.5 \mathrm{~T}$, but not identified: ${ }^{34}$ five are reported here to fields of $30 \mathrm{~T}$ (Fig. 5).

Is this new series transitions to another excited state of $\mathrm{Zn}$ impurity? If so, the level is extremely shallow, $43 \mathrm{meV}$ compared with $45.5-50 \mathrm{meV}$ binding energy (Table I). In particular, it is not the lines $C, 1$, or 2 , as it corresponds to none of

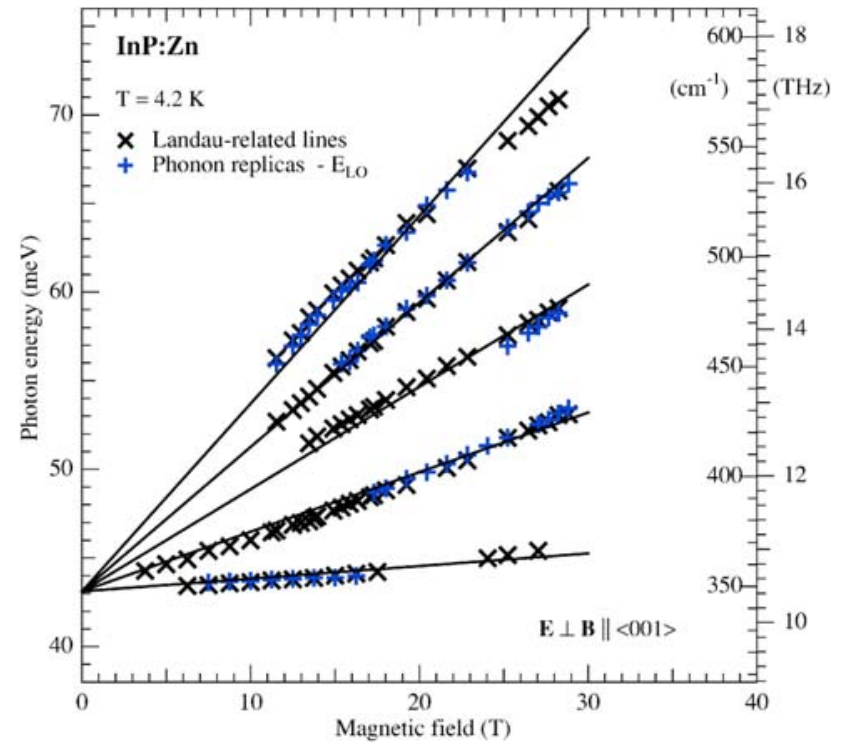

FIG. 7. (Color online) Landau-related series and their phonon replicas. The offset of the two series determines $\omega_{\mathrm{LO}}$, the intercept determines $E_{A}$, and the slope determines $m_{h}^{*}$.

these in energy. ${ }^{10,37}$ It cannot be the $2 P_{1 / 2}\left(1 \Gamma_{6}^{-}\right)$state (see Table II); this splits to only four components in field. ${ }^{31}$ So we rule out an excited state of $\mathrm{Zn}$ as the origin of the series. It may be thought the series originates from another impurity in InP. This is unlikely, as the energy does not correspond to any reported impurity: $\mathrm{Be}, \mathrm{Mg}, \mathrm{C}$, and $\mathrm{Ca}$ have smaller ionization energies, ${ }^{4}$ the $\mathrm{Cd}$ lines are nearby but do not match, ${ }^{10}$ $\mathrm{Si}$ and $\mathrm{Ge}$ are far deeper. ${ }^{4}$

In view of the series not being associated with bound states, and the repeated spacing in energy with field, we attribute it to free-hole Landau-related levels. These have been studied extensively in Ge, but not in III-Vs.

A further fascinating feature arises in high fields: phonon replicas of the Landau series. Before discussing the data, two limitations of the present experiments will be pointed out. First, absorption in a vacuum window prevents useful data being taken from $88-92 \mathrm{meV}$, and the features all move through this region as field is applied. Second, spectra were recorded only to $112 \mathrm{meV}$. The highest-energy feature moves beyond this before the highest field is reached. To bring out most clearly the weaker features, spectra taken at field were ratioed with the zero-field spectrum. Such ratios are shown in Fig. 6 for 15.4, 22.8, and 28.8 T. At 15.4 T, only components (c) and (d) are evident. At $22.8 \mathrm{~T}$, components (a), (b), (c), and (d) are all observed. By $28.8 \mathrm{~T}$, component (d) has moved out of the accessible energy range.

The energies of the Landau lines and their replicas are plotted against magnetic field in Fig. 7. The correspondence is excellent. Unlike for the $G$ and $D$ lines, there is no offset. The energy difference between the two series directly gives the phonon energy which we deduce from our data to be $\hbar \omega_{\mathrm{LO}}=43.1 \pm 0.3 \mathrm{meV}$. From the intercept we estimate the ionization energy of $\mathrm{Zn}$ in InP to be $E_{A}=43.0 \pm 0.8 \mathrm{meV}$. The slopes allow the hole effective mass to be determined, $m_{h}^{*}=0.59 \pm 0.02$. 


\section{CONCLUSION}

The observation of Landau-related lines in the absorption spectrum of InP:Zn allows the direct experimental determination of $E_{A}$ and $m_{h}^{*}$ and so $\gamma_{1}$. Observation of phonon replicas of these allows determination of $\omega_{\mathrm{LO}}$. The relaxation of selection rules for phonon replicas (Fano resonances) of bound-to-bound hole transitions relative to the Lyman series allows more reliable, and in some cases the only, values for the $g$ factors of the ground and excited states of the acceptor to be determined.

\section{ACKNOWLEDGMENTS}

This work was supported by the Australian Research Council and the University of Wollongong. Part of the work was performed at the National High Magnetic Field Laboratory, which was supported by NSF Cooperative Agreement No. DMR-9527035 and by the State of Florida. We thank Dr. G. Irmer (Technische Universität Bergakademie Frieberg) for providing the samples used in this investigation.
${ }^{1}$ A. Baldereschi and N. O. Lipari, Phys. Rev. B 8, 2697 (1973).

${ }^{2}$ A. Baldereschi and N. O. Lipari, Phys. Rev. B 9, 1525 (1974).

${ }^{3}$ A. Baldereschi and N. O. Lipari, J. Lumin. 12/13, 489 (1973).

${ }^{4}$ H. Wang and A.-B. Chen, Phys. Rev. B 63, 125212 (2001).

${ }^{5}$ E. Kubota, A. Katsui, and K. Sugii, J. Appl. Phys. 59, 3841 (1986).

${ }^{6}$ B. J. Skromme, G. E. Stillman, J. D. Oberstar, and S. S. Chan, J. Electron. Mater. 13, 463 (1984).

${ }^{7}$ P. J. Dean, D. J. Robbins, and S. G. Bishop, J. Phys. C 12, 5567 (1979).

${ }^{8}$ E. Kubota, Y. Omori, and K. Sugii, J. Appl. Phys. 55, 3779 (1984).

${ }^{9}$ E. W. Williams, W. Elder, M. G. Astles, M. Webb, J. B. Mullin, B. Straughan, and P. J. Tufton, J. Electrochem. Soc. 120, 1741 (1973).

${ }^{10}$ M. Wenzel, G. Irmer, and J. Monecke, Solid State Commun. 104, 371 (1997).

${ }^{11}$ W. O. G. Schmitt, E. Bangert, and G. Landwehr, J. Phys.: Condens. Matter 3, 6789 (1991).

${ }^{12}$ P. J. Dean, D. J. Robbins, and S. G. Bishop, Solid State Commun. 32, 379 (1979)

${ }^{13}$ U. Fano, Phys. Rev. 124, 1866 (1961).

${ }^{14}$ G. D. Watkins and W. B. Fowler, Phys. Rev. B 16, 4524 (1977).

${ }^{15}$ R. Baron, M. H. Young, and T. C. McGill, Solid State Commun. 47, 167 (1983)

${ }^{16}$ Y.-C. Chang and T. C. McGill, Solid State Commun. 47, 171 (1983).

${ }^{17}$ E. Janzén, G. Grossman, R. Stedman, and H. G. Grimmeiss, Phys. Rev. B 31, 8000 (1985).

${ }^{18}$ M. Kleverman, J. Olajos, and H. G. Grimmeiss, Phys. Rev. B 35, 4093 (1987).

${ }^{19}$ M. Kleverman, J. Olajos, and H. G. Grimmeiss, Phys. Rev. B 37, 2613 (1988).

${ }^{20}$ M. Kleverman, J.-O. Fornell, J. Olajos, H. G. Grimmeiss, and J.
L. Lindström, Phys. Rev. B 37, 10199 (1988).

${ }^{21}$ M. Suezawa, A. Kasuya, K. Sumino, and Y. Nishina, J. Phys. Soc. Jpn. 57, 4021 (1988).

22 J. Olajos, M. Kleverman, and H. G. Grimmeiss, Phys. Rev. B 38, 10633 (1988)

${ }^{23}$ A. W. Simonian, A. B. Sproul, Z. Shi, and E. Gauja, Phys. Rev. B 52, 5672 (1995).

${ }^{24}$ G. Piao, R. A. Lewis, and P. Fisher, Phys. Rev. B 54, 1741 (1996).

${ }^{25}$ H. G. Grimmeiss, L. Montelius, and K. Larsson, Phys. Rev. B 37, 6916 (1988).

${ }^{26}$ G. Piao, P. Fisher, and R. A. Lewis, Phys. Rev. B 61, 7466 (2000).

${ }^{27}$ M. Linnarsson, E. Janzén, B. Monemar, M. Kleverman, and A. Thilderkvist, Phys. Rev. B 55, 6938 (1997).

${ }^{28}$ H. Nakata, K. Yamada, and T. Ohyama, Phys. Rev. B 60, 13269 (1999).

${ }^{29}$ G. Piao, R. A. Lewis, and P. Fisher, Solid State Commun. 75, 835 (1990).

${ }^{30}$ P. Lawaetz, Phys. Rev. B 4, 3460 (1971).

${ }^{31}$ A. K. Bhattacharjee and S. Rodriguez, Phys. Rev. B 6, 3836 (1972).

${ }^{32}$ T. L. Linnik and V. I. Sheka, Phys. Solid State 41, 1425 (1999).

${ }^{33}$ R. A. Lewis, Y.-J. Wang, and M. Henini, Phys. Rev. B 67, 235204 (2003).

${ }^{34}$ R. A. Lewis and Y. J. Wang, Solid State Commun. 26, 275 (2003).

${ }^{35} \mathrm{G}$. Piao, R. A. Lewis, and P. Fisher, in The 21st International Conference on the Physics of Semiconductors, edited by P. Jiang and H. Z. Zheng (World Scientific, Singapore, 1992), pp. 16091612.

${ }^{36}$ R. L. Causley and R. A. Lewis, Physica B 302-303, 327 (2001).

${ }^{37}$ G. Irmer, M. Wenzel, and J. Monecke, Phys. Status Solidi B 210, 347 (1998). 